\title{
Short-wavelength intersubband absorption in strain compensated InGaAs/AIAs quantum well structures grown on InP
}

\author{
N. Georgiev, ${ }^{\text {a) }}$ T. Dekorsy, F. Eichhorn, and M. Helm \\ Institute of Ion Beam Physics and Materials Research, Forschungszentrum Rossendorf, P.O. Box 510119, \\ D-01314 Dresden, Germany \\ M. P. Semtsiv and W. T. Masselink \\ Department of Physics, Humboldt-University Berlin, Invalidenstrasse 110, D-10115 Berlin, Germany
}

\begin{abstract}
We have studied intersubband absorption in strain compensated $\operatorname{In}_{x} \mathrm{Ga}_{1-x} \mathrm{As} / \mathrm{AlAs} / \mathrm{In}_{y} \mathrm{Al}_{1-y} \mathrm{As}$ multiple quantum wells and superlattices grown on InP. X-ray diffraction shows that the layers are pseudomorphically strained and exhibit slight compositional grading of the interfaces. Owing to the high AlAs barriers, the intersubband absorption can be tailored to wavelengths shorter than $2 \mu \mathrm{m}$. In some samples, a small, but non-negligible absorption is also observed with $s$-polarized light.
\end{abstract}

Intersubband transitions are now routinely used for optoelectronic devices such as quantum cascade lasers (QCL) and quantum well infrared detectors in the midinfrared (wavelength $\lambda=3-20 \mu \mathrm{m}$ ). ${ }^{1}$ Recently also a QCL in the far infrared, at $70 \mu \mathrm{m}$, has been reported. ${ }^{2}$ Yet there is considerable interest to extend applications of intersubband transitions also to the near infrared $(<3 \mu \mathrm{m})$ and in particular to the $1.55 \mu \mathrm{m}$ communication wavelength region. Such devices would offer very fast switching and modulation frequencies ${ }^{3}$ due to the fast intersubband relaxation in the subpicosecond regime.

In the past few years short-wavelength intersubband absorption has been demonstrated in several material systems, such as $\mathrm{GaN} / \mathrm{AlGaN}^{4-6}$ or $\mathrm{ZnSe} / \mathrm{BeTe}^{7}$ Yet it would still be preferred to employ common III-V technology based on GaAs or InP. In the GaAs/AlGaAs system the band offset at the $\Gamma$ point can be as large as $1.0 \mathrm{eV}$ for pure AlAs, but the $\mathrm{X}$ states in AlAs are much lower and can even drop below the GaAs quantum well state in narrow quantum wells (QW). This can be partly remedied by using strained InGaAs, which increases the band offset and also lowers the QW states. Short-wavelength intersubband absorption in this system has been reported by several groups and the $\Gamma-\mathrm{X}$ crossover has been observed. ${ }^{8-10}$ On the other hand, lattice matched InGaAs/InAlAs on InP has a conduction band offset of $0.52 \mathrm{eV}$. Larger offsets can only be achieved in strained structures, which have for instance been employed in the shortest-wavelength QCL to date $(\lambda=3.5 \mu \mathrm{m}){ }^{11} \mathrm{InGaAs}$ QWs on InP with pure AlAs barriers have only been investigated by one group. ${ }^{12}$ They observed absorption wavelengths as short as $1.55 \mu \mathrm{m}$ as well as a strong, redshifted intersubband absorption in the normally forbidden $s$-polarization. This has been an issue of extensive discussion in the literature, ${ }^{13}$ because other groups were able to observe only weak $s$-polarized signals. ${ }^{14}$ An alternative heterosystem lattice matched to InP is $\operatorname{In}_{0.53} \mathrm{Ga}_{0.47} \mathrm{As} / \mathrm{AlAs}_{0.56} \mathrm{Sb}_{0.44}$, which

${ }^{a)}$ Electronic mail: n.georgiev@fz-rossendorf.de has a very high band offset and is also promising for shortwavelength applications. ${ }^{15}$

In the present work we explore the potential of the InGaAs/AlAs system grown pseudomorphically on InP substrates for short-wavelength intersubband transitions. Our strategy is to use InGaAs quantum wells with a large In content, which are compressively strained: this provides deep QWs, a $\Gamma$-like ground state, and helps to compensatetogether with a slightly compressively strained $\operatorname{In}_{0.55} \mathrm{Al}_{0.45} \mathrm{As}$ buffer-the strain from the tensile strained AlAs barriers. When good overall strain compensation is achieved, thick multilayer structures can be grown pseudomorphically.

All samples were grown by gas-source molecular-beam epitaxy (GSMBE) on semi-insulating InP (001) substrates. To provide a smooth growth front a $200 \mathrm{~nm}$ undoped InP buffer was grown first at $465^{\circ} \mathrm{C}$ followed by an undoped $\mathrm{In}_{0.55} \mathrm{Al}_{0.45} \mathrm{As}$ buffer $\left(480{ }^{\circ} \mathrm{C}\right)$ and the $\mathrm{QW}$ structures $\left(400{ }^{\circ} \mathrm{C}\right)$. Two types of structures were grown, namely multiquantum wells (MQWs) and short-period superlattices (SLs). The MQWs consist of 30 periods of $\operatorname{In}_{0.7} \mathrm{Ga}_{0.3}$ As wells with various thicknesses (see later) confined by 1.5- or 2.0nm-thick AlAs barriers. A 20 -nm-thick $\operatorname{In}_{0.55} \mathrm{Al}_{0.45}$ As layer is inserted between each period to compensate the large tensile strain caused by the AlAs layers. The SLs consist of 40 pairs of $\operatorname{In}_{0.7} \mathrm{Ga}_{0.3} \mathrm{As}(4.2$ or $3.3 \mathrm{~nm})$ and AlAs $(1.2 \mathrm{~nm})$. In all cases the $\mathrm{In}_{0.7} \mathrm{Ga}_{0.3} \mathrm{As} \mathrm{QW}$ layers are $\mathrm{Si}$ doped (for details see later). A sketch of the conduction band alignment of the MQWs is shown in the inset of Fig. 2. It should be noted that due to the high In content in the wells, the QW ground states are well below the $\mathrm{X}$ states in the barriers. According to Van de Walle's model-solid theory, ${ }^{16}$ the conduction band offset between wells and barriers can be estimated to $1.23 \mathrm{eV}$. Considering only the high In content in the wells, one would expect an even higher offset, but part of it is compensated by the strain-induced shift of the band edges.

High-resolution x-ray diffraction (XRD) was performed at the European Synchrotron Radiation Facility in Grenoble (France). The diffraction pattern near the (004) reflex of a MQW sample with 7 monolayers (ML) thick $\operatorname{In}_{0.7} \mathrm{Ga}_{0.3}$ As QWs is presented in Fig. 1. Using a dynamical simulation, 


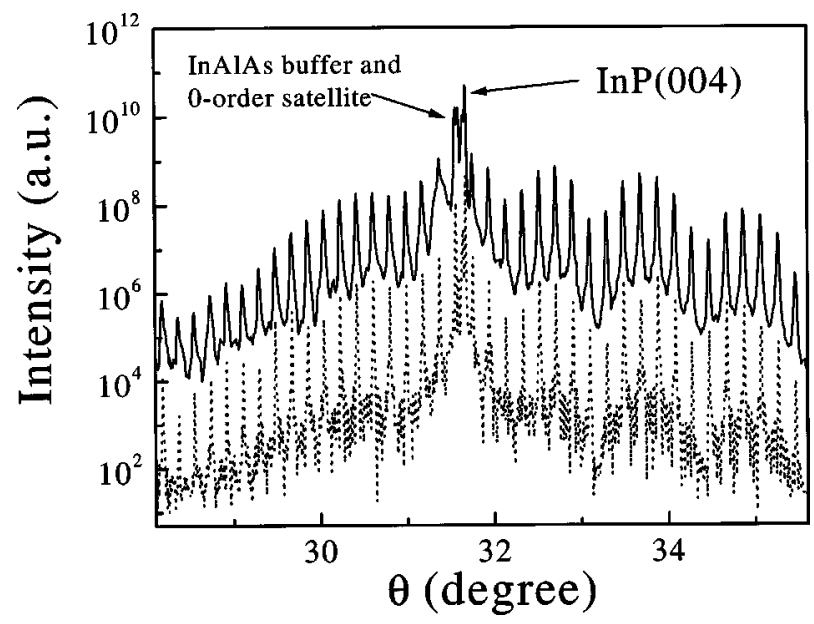

FIG. 1. XRD pattern of the $7 \mathrm{ML} \mathrm{In}_{0.7} \mathrm{Ga}_{0.3} \mathrm{As} / \mathrm{AlAs} / \mathrm{In}_{0.55} \mathrm{Al}_{0.45} \mathrm{As} \mathrm{MQW}$ around the InP (004) reflex. Experimental data (solid line) and simulation (dotted line) using a model that includes interfacial compositional grading.

the two peaks very close to the substrate peak (lower-angle side) can be identified as zero-order MQW peak and as originating from the $\mathrm{In}_{0.55} \mathrm{Al}_{0.45} \mathrm{As}$ buffer, respectively. The tiny splitting indicates nearly perfect overall strain compensation, to better than $0.2 \%$. The best fit (dotted line), however, is achieved by assuming interfaces which are not sharp, but rather compositionally graded by 1-2 ML. In addition, reciprocal space maps at the (224) and (115) reflections were employed to verify that the QW structures were grown fully pseudomorphic and no strain relaxation by generation of misfit dislocations occurred.

The intersubband absorption spectra were measured at room temperature with a Fourier-transform infrared spectrometer using a multiple-reflection waveguide geometry fabricated by polishing two end facets at an $45^{\circ}$ angle. Figure 2 shows the transmission spectra of three MQW samples with well thicknesses of $2.8,2.2$, and $1.9 \mathrm{~nm}$, corresponding to about 9,7 , and $6 \mathrm{ML}$, respectively. Plotted is the ratio of the $p$ - and $s$-polarized transmission, further normalized to the same ratio of an undoped InP substrate. The absorption peaks are observed at $0.58,0.66$, and $0.72 \mathrm{eV}$, respectively, the latter two corresponding to wavelengths shorter than 2 $\mu \mathrm{m}$. All experimental peaks occur at energies which are

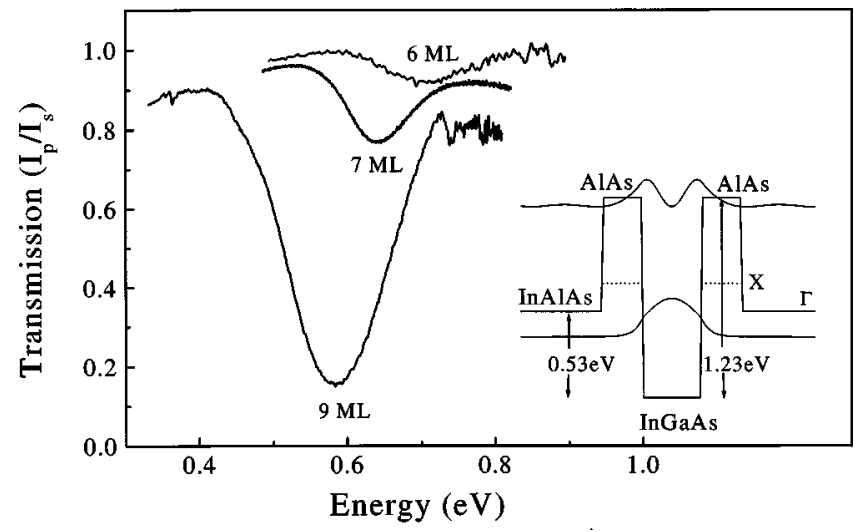

FIG. 2. Transmission spectra of the $\operatorname{In}_{0.7} \mathrm{Ga}_{0.3} \mathrm{As} / \mathrm{AlAs} / \mathrm{In}_{0.55} \mathrm{Al}_{0.45} \mathrm{As} \mathrm{MQWs}$ with different InGaAs well widths. The inset shows the schematic conduction band diagram of the QW structure and calculated wave functions for the sample with 7-ML-thick wells.

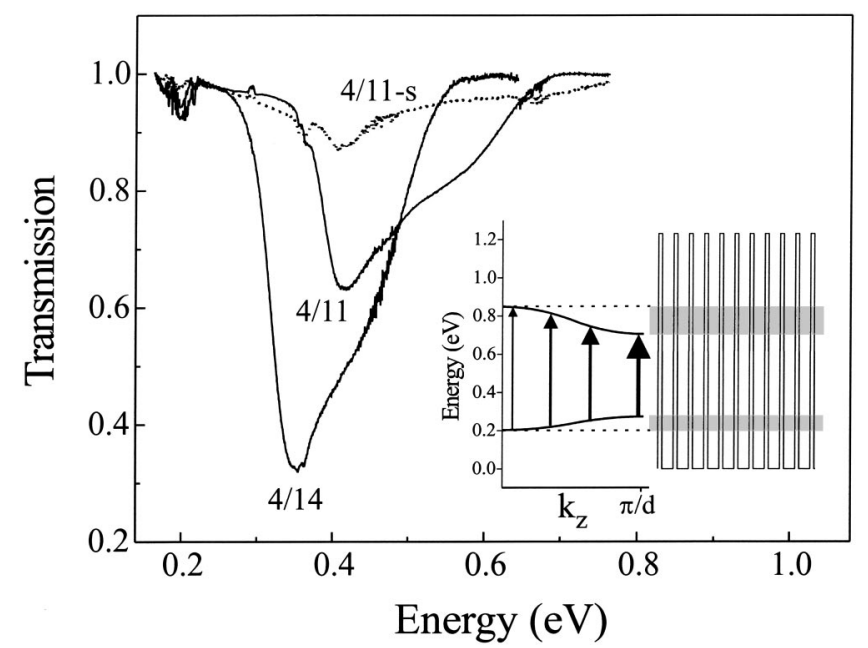

FIG. 3. Transmission spectra of the two $\operatorname{In}_{0.7} \mathrm{Ga}_{0.3} \mathrm{As} / \mathrm{AlAs}$ superlattices with 14 and 11 MLs well widths, respectively. In addition, the pure $s$-polarized spectrum is plotted for the 4/11 SL (dotted). The inset shows the conduction band diagram together with the calculated miniband dispersion for the $4 / 11$ ML SL. The thickness of the arrows schematically indicates the variation of the oscillator strength along the wave vector $k_{z}$.

about $10 \%-15 \%$ too low, when compared to a calculation in the framework of the effective mass approximation including nonparabolicity and strain-modified band edges. ${ }^{16}$ If we, however, assume a modified QW potential with a 1 ML-thick interface layer having an intermediate composition between well and barrier layers, we can reproduce the experimental values of the peak energies within a few percent. This is supported by the XRD measurements, which yield the best fit with such modified interface layers (Fig. 1) as described earlier. Although low-temperature growth of the QW structures suppresses In segregation and interdiffusion across the heterointerfaces, some short-scale interface roughness during the growth of heavily strained layers may still develop, ${ }^{17}$ which effectively also yields a thin interface layer with intermediate composition.

Next we focus on the absorption strengths and widths. The observed absorption linewidths are relatively large (full width of $120-170 \mathrm{meV}$, i.e., relative linewidth of $15 \%-$ $20 \%$ ), an observation which is quite typical for shortwavelength intersubband absorption. ${ }^{8}$ It is caused by interface roughness and well thickness fluctuations, which have a significant influence in thin QWs, and also partly by nonparabolicity in connection with the high doping. The absorption strength should be proportional to the areal electron density. We note that the 9 and 6 ML samples are nominally doped $1 \times 10^{19} \mathrm{~cm}^{-3}$ in the wells, whereas the $7 \mathrm{ML}$ sample only $2 \times 10^{18} \mathrm{~cm}^{-3}$. Thus, the absorption decrease from the 9 to the $7 \mathrm{ML}$ sample appears reasonable; however, the absorption of the 6 ML sample is by far too weak and cannot be explained by simple means (similar observations have been made in Ref. 8). A partial reason may be a smaller oscillator strength since for this sample the upper state is already in the continuum above the barriers (the inset in Fig. 2 shows the 7 ML sample with still marginal confinement); the main cause why the carriers do not participate in the absorption process, remains unclear however.

In Fig. 3 the transmission spectra of the two superlattice samples are presented. They have the same barrier width (1.2 
$\mathrm{nm}$ or $4 \mathrm{ML}$ ), but different well thicknesses of $4.2 \mathrm{~nm}$ and $3.3 \mathrm{~nm}$ (14 and $11 \mathrm{ML}$ ), respectively, and are doped in the wells with $n=2 \times 10^{18} \mathrm{~cm}^{-3}$. Plotted is again the ratio of $p$ to $s$-polarized signals normalized to a substrate signal as described earlier. The spectra clearly exhibit the asymmetric shape which is typical for strongly coupled superlattices, due to the variation of the oscillator strength along the SL wave vector $k_{z} \cdot{ }^{18}$ The peak and the shoulder correspond to the transitions at the edge $\left(k_{z}=\pi / d\right)$ and the center $\left(k_{z}=0\right)$ of the mini-Brillouin zone, respectively (see inset of Fig. 3). The transition at $k_{z}=\pi / d$ can be observed, because the first miniband is fully occupied due to the high doping density. Its position corresponds to the minigap of the SL as illustrated in the inset, where the calculated miniband dispersion is shown for the 4/11 ML superlattice. The peak positions ( 0.355 and $0.415 \mathrm{eV}$ for the $4 / 14$ and $4 / 11 \mathrm{ML}$ superlattice, respectively) agree well $(<5 \%)$ with the calculated values using graded interfaces. Due to their larger well width, the influence of the interface grading for the SLs is, however, not as large as for the MQW samples. Also in contrast to the MQW spectra (Fig. 2), a significant contribution to the total linewidth in the SL spectra (Fig. 3) comes from the intrinsic width of the minibands. ${ }^{18}$ Note that for the $4 / 11$ ML superlattice the absorption extends up to nearly $0.7 \mathrm{eV}$, corresponding to a wavelength of roughly $1.8 \mu \mathrm{m}$.

An additional, third spectrum is displayed in Fig. 3 (dotted line). It is the pure $s$-polarized signal of the $4 / 11 \mathrm{ML}$ superlattice, divided by the $s$-polarized signal through an undoped substrate. It appears rather convincing that there is some finite intersubband absorption remaining in $s$-polarization, $10 \%-20 \%$ the size of the $p$-polarized absorption (the sharp features at $0.37 \mathrm{eV}$ stem from organic molecules probably on the sample holder). In some of the other samples, there is an indication of similar behavior (not shown), but it cannot always be unambiguously separated from the background. These observations do not support the previous work of Smet et al. ${ }^{12}$ who observed a comparable size of $s$ and $p$ absorption, but are rather consistent with later studies by Liu, ${ }^{14}$ who showed $3 \% s$-polarized absorption in $\mathrm{In}_{0.1} \mathrm{Ga}_{0.9} \mathrm{As}$ QWs. Theoretical work predicts some small $s$-polarized absorption resulting from $\mathbf{k} \cdot \mathbf{p}$ coupling to higher bands. ${ }^{19}$ Presently there are no detailed calculations for the exact structures investigated here, but due to their high In content and the large strain it appears not unreasonable to assume a modest relaxation of the intersubband selection rule, resulting in a "forbidden" absorption strength as observed in the present work.
In conclusion, we have studied intersubband absorption in highly strained, but strain compensated InGaAs/AlAs multiquantum well and superlattice structures, with absorption wavelengths below $2 \mu \mathrm{m}$. Small $s$-polarized absorption is also observed, which, however, does not appear to contradict theoretical considerations.

This work was supported by the Deutsche Forschungsgemeinschaft (DFG) within the Forschergruppe FOR 394. The authors are grateful to A. Bauer for help with the XRD measurements.

${ }^{1}$ Semiconductors and Semimetals, edited by H. C. Liu and F. Capasso (Academic, New York, 2000), Vols. 62 and 66.

${ }^{2}$ R. Koehler, A. Tredicucci, F. Beltram, H. E. Beere, E. H. Linfield, A. G. Davies, D. A. Ritchie, R. C. Iotti, and F. Rossi, Nature (London) 417, 156 (2002).

${ }^{3}$ T. Asano, M. Tamura, S. Yoshizawa, and S. Noda, Appl. Phys. Lett. 77, 19 (2000); T. Akiyama, N. Georgiev, T. Mozume, A. V. Gopal, and O. Wada, IEEE Photonics Technol. Lett. 14, 495 (2002).

${ }^{4}$ C. Gmachl, H. M. Ng, S.-N. G. Chu, and A. Y. Cho, Appl. Phys. Lett. 77, 3722 (2000); J. D. Heber, C. Gmachl, H. M. Ng, and A. Y. Cho, ibid. 81, 1237 (2002).

${ }^{5}$ N. Iizuka, K. Kaneko, and N. Suzuki, Appl. Phys. Lett. 81, 1803 (2002).

${ }^{6}$ K. Kishino, A. Kikuchi, H. Kanazawa, and T. Tachibana, Appl. Phys. Lett. 81, 1234 (2002).

${ }^{7}$ R. Akimoto, K. Akita, F. Sasaki, and T. Hasama, Appl. Phys. Lett. 81, 2998 (2002)

${ }^{8}$ H. C. Chui, E. L. Martinet, M. M. Fejer, and J. S. Harris, Jr., Appl. Phys. Lett. 64, 736 (1994); B. Sung, H. C. Chui, M. M. Fejer, and J. S. Harris, Jr., Electron. Lett. 33, 818 (1997).

${ }^{9}$ J. M. Jancu, V. Pellegrini, R. Colombelli, F. Beltram, B. Mueller, L. Sorba, and A. Franciosi, Appl. Phys. Lett. 73, 2621 (1998).

${ }^{10}$ T. Asano, S. Noda, T. Abe, and A. Sasaki, J. Appl. Phys. 82, 3385 (1997).

${ }^{11}$ J. Faist, F. Capasso, D. L. Sivco, A. L. Hutchinson, S.-N. G. Chu, and A. Y. Cho, Appl. Phys. Lett. 72, 680 (1998).

${ }^{12}$ J. H. Smet, L. H. Peng, Y. Hirayama, and C. G. Fonstad, Appl. Phys. Lett. 64, 986 (1994), and references therein.

${ }^{13}$ L. C. Lew Yan Voon, M. Willatzen, M. Cardona, and I. R. Ram-Mohan, J. Appl. Phys. 80, 600 (1996), and references therein.

${ }^{14}$ H. C. Liu, M. Buchanan, and Z. R. Wasilewski, Appl. Phys. Lett. 72, 1682 (1998), and references therein.

${ }^{15}$ A. Neogi, H. Yoshida, T. Mozume, N. Georgiev, T. Akiyama, and O. Wada, Physica E (Amsterdam) 7, 183 (2000); A. V. Gopal, H. Yoshida, T. Simoyama, N. Georgiev, T. Mozume, and H. Ishikawa, IEEE J. Quantum Electron. 39, 299 (2003).

${ }^{16}$ C. G. Van de Walle, Phys. Rev. B 39, 1871 (1989).

${ }^{17}$ V. Drouot, M. Gendry, C. Santinelli, X. Letart, J. Tardy, P. Viktorovitch, G. Holliger, M. Ambri, and M. Pitaval, IEEE Trans. Electron Devices 43, 1326 (1996).

${ }^{18}$ M. Helm, W. Hilber, T. Fromherz, F. M. Peeters, K. Alavi, and R. N. Pathak, Phys. Rev. B 48, 1601 (1993); M. Helm, Semicond. Sci. Technol. 10, 557 (1995).

${ }^{19}$ R. Q. Yang, J. M. Xu, and M. Sweeny, Phys. Rev. B 50, 7474 (1994); R. J. Warburton, C. Gauer, A. Wixforth, J. P. Kotthaus, B. Brar, and H. Kroemer, ibid. 53, 7903 (1996); M. E. Flatté, P. M. Young, L.-H. Peng, and H. Ehrenreich, ibid. 53, 1963 (1996). 